\title{
Índice de autores 2019
}

La Revista de la Asociación Argentina de Ortopedia y Traumatología agradece a los siguientes autores por habernos elegido para publicar sus trabajos. Esperamos seguir contando con su valiosa colaboración.

Allende B. L. 320

Allende G. J. 27

Allende Nores C. A.

Amaya M. $99 ; 285 ; 353$

Ameriso N. 236

Anchuela Ocaña J. J. 252

Andreozzi R. .. 361

Arcos A. 35

Argüelles S. 372

Arzac Ulla I. R. 342

Autorino C.M. 129; 336

Balmaceda M. M. 178

Barasatián P. 35; 236

Barla J. D. 372

Barriga-Martín A. 242

Bassani J. 273

Bazán P. L. .. 149

Belluschi G. 208

Bendersky M. 35

Bennice J. ... 427

Berenstein M. .. 90

Bersusky S.E $84 ; 224$

Besse M.

Bidolegui F. 15

Bordón M. E. 27

Boretto J. G. 46; 90; 143; 427

Borri Á. E. 208

Brandariz R. .. 90

Buttaro M

Cáceres-Palou E. .. 393

Caloia M.

Camino Willhuber G.

Cancer Castillo D.

Capomassi M.
Carabelli G.

242

Carrioli G. G. 216; 406

Caruso J. C. 386

Castellini J. .. 75

Catapano F. 178

Chuliá Carrasco V. .................................................................. 260

Ciccioli N. M. ...................................................................... 208

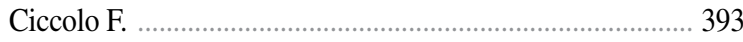

Cid Casteulani A. ............................................................... 15

Cock Atehortua J. G. ......................................................... 265

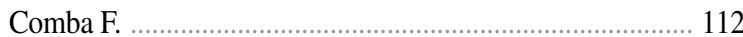

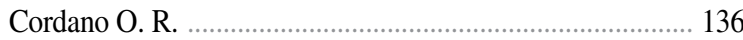

Coria H. ......................................................................... 252

Corrales Pinto J. .............................................................. 208

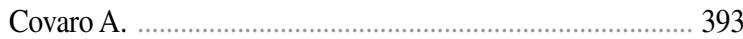

Cubecino A. E. ................................................................ 420

Dalmasso M. L. .............................................................. 3; 80

De Carli P. …............................................... 46; 90; 143; 427

De Cicco F. L. ........................................................................ 242

del Sel H. ........................................................................ 328

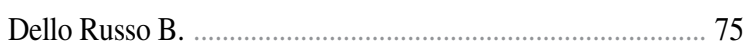

Donndorff A. G. .................................................... 46; 427

Eluani M. S. .................................................................. 320

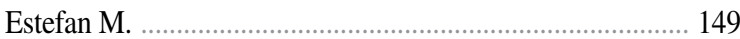

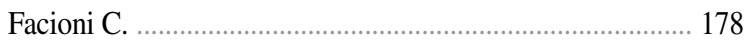

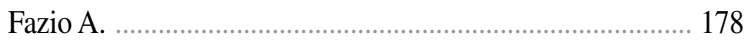

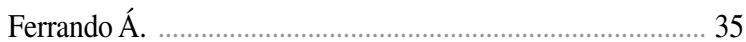

Francheri Wilson I. A. ..................................................... 224

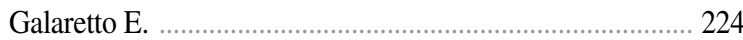

Gallucci G. L. ........................................... 46; 90; 143; 427

Garabano G. ....................................................... 15; 328

Garcia de Frutos A. ………………..................................... 393

Garfinkel I. G. ...................................................... 216; 406

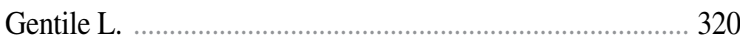


Ghisi J. P. $3 ; 80 ; 86 ; 188 ; 202 ; 309 ; 314 ; 434$

Gobbi E. A. 70

Gómez Barbero P. 260

Gomez Cano J. .. 208

Gómez D. J. 15

González G. A. 105

Gorodischer T. 143

Grau Núñez L.

Gruenberg M. 149

Guerrini A. F.

Guimbard Pérez J. H. 105; 273

Gutiérrez R. 70

Herrera H. S. ... 75

Iglesias $\mathrm{S}$. ... 353

Isart Torruela $\mathrm{A}$ 393

Juarez A. B. 320

Kido G. 149

Kobrinsky P. ... 122

Lamanna A. $86 ; 188$

Lanari Subiaur F. J. .... 70

Lauritto D. .. 236

Lobos Centeno E. A. ... 285

López P. 285

Loterzo L.G. $155 ; 313$

Mangupli M. 285

Manzone P. 197; 437

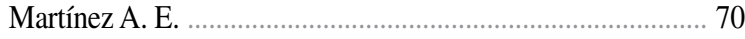

Martínez G. M. 361

Masaragian $\mathrm{H}$. 252

Masquijo J. J. 27

Medina $\mathrm{M}$. 208

Mereles M. .. 149

Minig M. 27

Mizdraji L. 252

Montero Vinces A. ............................................................. 15

Mounier C.M. 70

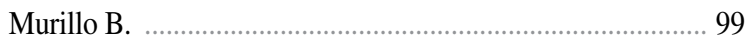

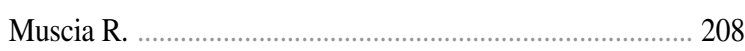

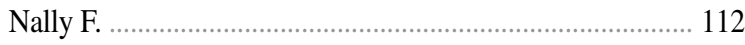

Noel M. A. 224

Oñativia J. I.

Ortiz N.
Parisi M. 178

Pascual-Garrido C. …………………………………............. 296

Patiño J. M. ..................................................................... 386

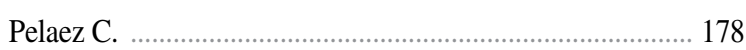

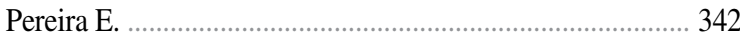

Pérez Alamino L. ............................................................ 328

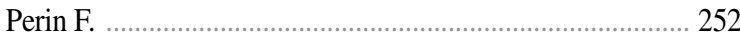

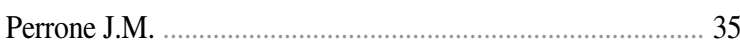

Pesciallo C. Á. .......................................................... 15; 328

Petracchi M. ..................................................................... 149

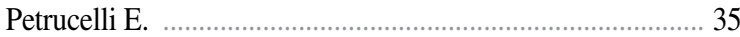

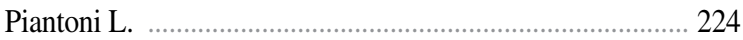

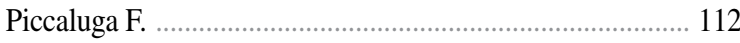

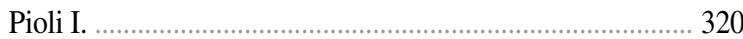

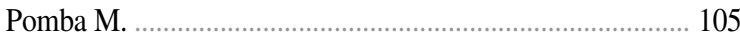

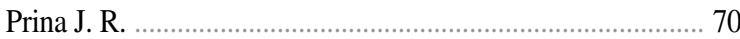

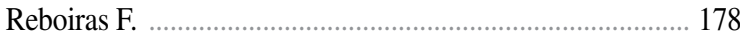

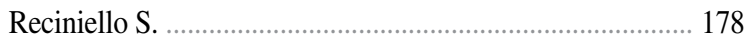

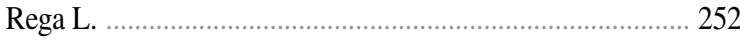

Rellán I. ................................................................. 46; 427

Remondino R.

Restrepo Noriega V. E. ......................................................... 265

Ricciardi D. O.

Ricciardi G. A. ..................................................... 216; 406

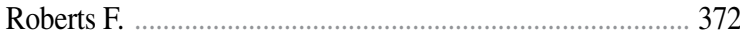

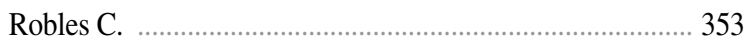

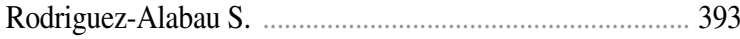

Rodríguez-Fontán F. ........................................................... 296

Rodríguez M. P. ……........................................................ 342

Rodríguez O. ............................................................... 99

Rodriguez Sammartino M. ................................................ 1

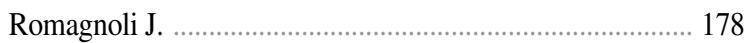

Romero-Muñoz L. M. .................................................... 273

Rosado Pardo J. A. .............................................................. 420

Rosales Anderica F. E. ....................................................... 236

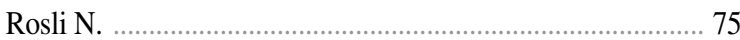

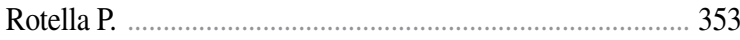

Salcedo R. ……............................................................... 112

Sánchez Villanueva F. J. ................................................ 8; 63

Sancineto C. F. ..................................................... 178; 242

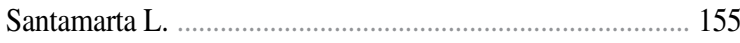

Santini Araujo M. G. .......................................................... 75 
Santodomingo J.

437

Sarmiento H.

Sarotto A.

Sartor F.

Sartori P. M.

Schneebeli G.

Sepúlveda Oviedo M.

Sieger T. C.

Slullitel P.A.

Sola C.

Soutullo J.

Suárez Villalobos P.A.

Tacus L.

Taype Zamboni D.

Tello C. A.

Torrano J. P.

Torres-Gómez A.

Trache A.
.. 35

420

178

372

86; 188

$8 ; 63$

361

112

149

342

$8 ; 63$

122

242

178; 224

178

27

$3 ; 80 ; 314 ; 434$
Ubierna-Garcés M. ............................................................. 393

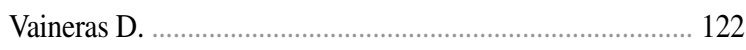

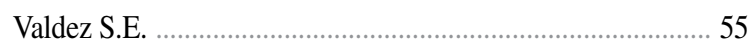

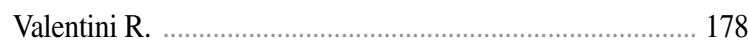

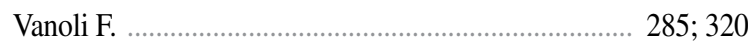

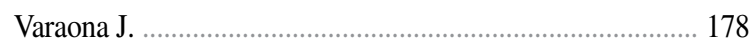

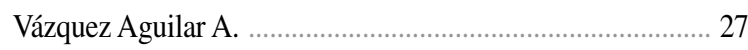

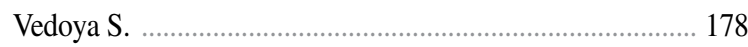

Vidondo M. E. ................................................... 202; 309

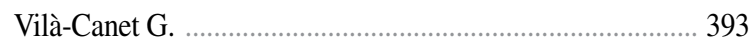

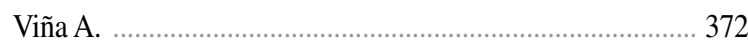

Vivas M. R. ……............................................... $75 ; 136$

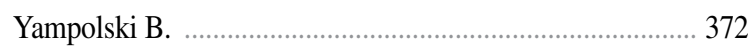

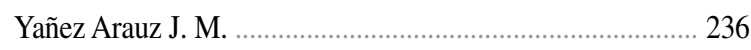

Yañez Arauz S. ................................................................... 236

Zaidenberg E. E. ............................................... 46; 90; 427

Zorzo Godes C. ............................................................ 361 